\title{
CONTINUOUSLY DIFFERENTIABLE MEANS
}

\author{
JUN ICHI FUJII, MASATOSHI FUJII, TAKESHI MIURA, \\ HIROYUKI TAKAGI, AND SIN-EI TAKAHASI
}

Received 3 March 2006; Revised 7 September 2006; Accepted 12 September 2006

We consider continuously differentiable means, say $C^{1}$-means. As for quasi-arithmetic means $Q_{f}\left(x_{1}, \ldots, x_{n}\right)$, we need an assumption that $f$ has no stationary points so that $Q_{f}$ might be continuously differentiable. Introducing quasi-weights for $C^{1}$-means would give a satisfactory explanation for the necessity of this assumption. As a typical example of a class of $C^{1}$-means, we observe that a skew power mean $M_{t}$ is a composition of power means if $t$ is an integer.

Copyright (C) 2006 Jun Ichi Fujii et al. This is an open access article distributed under the Creative Commons Attribution License, which permits unrestricted use, distribution, and reproduction in any medium, provided the original work is properly cited.

\section{Introduction}

Let $M\left(x_{1}, \ldots, x_{n}\right)$ be a continuously differentiable $n$-variable positive function on $(0, \infty)^{n}$. Then, throughout this paper, $M$ is called a continuously differentiable mean, or shortly $C^{1}$-mean if $M$ satisfies

(i) $M$ is monotone increasing in each term;

(ii) $M(a, \ldots, a)=a$ for all positive numbers $a$.

A mean $M$ is called homogeneous if $M$ satisfies

$$
M\left(a x_{1}, \ldots, a x_{n}\right)=a M\left(x_{1}, \ldots, x_{n}\right)
$$

for all $a, x_{k}>0$. Almost all classical means are homogeneous $C^{1}$-ones. The Kubo-Ando (operator) means in [6] and chaotic ones in [2] are $C^{1}$-means. Here note that (numerical) Kubo-Ando means $K_{f}(a, b)$ are defined by

$$
K_{f}(a, b)=a f\left(\frac{b}{a}\right)
$$

Hindawi Publishing Corporation Journal of Inequalities and Applications Volume 2006, Article ID 75941, Pages 1-15

DOI 10.1155/JIA/2006/75941 
for positive operator monotone functions $f$, which form a special class of numerical means.

Let $f$ be a continuously differentiable monotone function on $(0, \infty)$ with no stationary points, that is, $f^{\prime}(x) \neq 0$ for all $x>0$. In this case, $f^{-1}$ is also continuously differentiable. Let $w=\left\{w_{k}\right\}$ be a weight, that is, a set of nonnegative numbers $w_{k}$ with $\sum_{k} w_{k}=1$. For such $f$ and a weight $w$, it follows that a quasi-arithmetic mean $Q_{f, w}$ defined by

$$
Q_{f, w}\left(x_{1}, \ldots, x_{n}\right)=f^{-1}\left(\sum_{k=1}^{n} w_{k} f\left(x_{k}\right)\right)
$$

is a typical $C^{1}$-mean. As we will see later in the next section, the assumption that $f$ has no stationary points is necessary for continuous differentiability. Our main interest in this paper is when integral functions

$$
M_{f, P}\left(x_{1}, \ldots, x_{n}\right)=f^{-1}\left(\int_{0}^{\infty} f(x) d P_{x_{1}, \ldots, x_{n}}(x)\right)
$$

are $C^{1}$-means, where $P_{x_{1}, \ldots, x_{n}}$ is a probability measure on $(0, \infty)$ for each $x_{k}$. Note that these functions differ from the continuous quasi-arithmetic means, cf. $[4,5]$, but they include the above discrete quasi-arithmetic ones $Q_{f, w}$. In fact, for a convex combination for Dirac measures $P_{x_{1}, \ldots, x_{n}}=\sum_{k=1}^{n} w_{k} \delta_{x_{k}}$, we have $M_{f, P}=Q_{f, w}$.

In this paper, we discuss continuous differentiability of such integral functions as means, and observe when $M_{f, P}$ is a $C^{1}$-mean, particularly as 2 -variable functions. Many mathematicians have been interested in means of positive numbers. But, even in a quasiarithmetic mean, odd properties appear as we will see in some examples later. We noticed that the key in this problem is continuous differentiability for means. So we discuss continuously differentiable means and give some classes of such means. Finally, we discuss skew power means including logarithmic one as a path of $C^{1}$-means.

\section{Quasi-weight for means}

Power means defined by (see [3])

$$
P_{r, w}\left(x_{1}, \ldots, x_{n}\right)=\left(\sum_{k=1}^{n} w_{k} x_{k}^{r}\right)^{1 / r}
$$

are quasi-arithmetic $C^{1}$-means. Note that only homogeneous quasi-arithmetic means are the power means, which is shown in [4]. Then, we have

$$
\frac{\partial P_{r, w}}{\partial x_{k}}(a, \ldots, a)=\frac{1}{r}\left(\sum_{k=1}^{n} w_{k} a^{r}\right)^{1 / r-1} \cdot r w_{k} a^{r-1}=w_{k}\left(a^{r}\right)^{1 / r-1} a^{r-1}=w_{k} .
$$

Moreover, we have the following property.

Lemma 2.1. All $C^{1}$-means $M$ satisty $\left(\partial M / \partial x_{k}\right)(a, \ldots, a) \geqq 0$ and $\sum_{k=1}^{n}\left(\partial M / \partial x_{k}\right)(a, \ldots, a)=$ 1 for all $a>0$. 
Jun Ichi Fujii et al. 3

Proof. It follows from (ii) that

$$
1=\frac{a+\varepsilon-a}{\varepsilon}=\lim _{\varepsilon \rightarrow 0} \frac{M(a+\varepsilon, \ldots, a+\varepsilon)-M(a, \ldots, a)}{\varepsilon}=\sum_{k=1}^{n} \frac{\partial M}{\partial x_{k}}(a, \ldots, a) .
$$

The assumption (i) implies $\left(\partial M / \partial x_{k}\right)(a, \ldots, a) \geqq 0$.

Thereby, we define a $k$ th quasi-weight $w(M)_{k}(a)$ for a $C^{1}$-mean $M$ at $a$ by (cf. [1])

$$
w(M)_{k}(a)=\frac{\partial M}{\partial x_{k}}(a, \ldots, a) .
$$

Note that it is a constant for all $a$ if $M$ is homogeneous-like power means. In fact,

$$
\begin{aligned}
w(M)_{1}(a) & =\lim _{\varepsilon \rightarrow 0} \frac{M(a+\varepsilon, a, \ldots, a)-M(a, \ldots, a)}{\varepsilon} \\
& =\lim _{\varepsilon \rightarrow 0} \frac{M(1+\varepsilon / a, 1, \ldots, 1)-M(1, \ldots, 1)}{\varepsilon / a}=w(M)_{1}(1) .
\end{aligned}
$$

Moreover, even for a nonhomogeneous case, it can be constant and coincides with the weight.

THeOREM 2.2. If $f$ has no stationary point, then the kth quasi-weight $w\left(Q_{f, w}\right)_{k}(a)$ of a quasi-arithmetic mean $Q_{f, w}$ is the kth weight $w_{k}$.

Proof. Note that $\left(\partial f \circ Q_{f, w} / \partial x_{k}\right)(a, \ldots, a)=w_{k} f^{\prime}(a)$. On the other hand, we have

$$
\frac{\partial f \circ Q_{f, w}}{\partial x_{k}}(a, \ldots, a)=f^{\prime}\left(Q_{f, w}(a, \ldots, a)\right) \frac{\partial Q_{f, w}}{\partial x_{k}}(a, \ldots, a)=f^{\prime}(a) w\left(Q_{f, w}\right)_{k}(a),
$$

and hence $w\left(Q_{f, w}\right)_{k}(a)=w_{k}$ by $f^{\prime}(a) \neq 0$.

When $f$ has a stationary point, the following example shows that $Q_{f, w}$ is not always a $C^{1}$-mean.

Example 2.3. Let $Q_{f}=Q_{f,\{1 / 2,1 / 2\}}$. For a fixed $a>0$, put $f(x)=(x-a)^{3}+a^{3}$. Then we have $f^{-1}(x)=\left(x-a^{3}\right)^{1 / 3}+a$,

$$
\begin{aligned}
Q_{f}(x, y) & =\left(\frac{(x-a)^{3}+(y-a)^{3}}{2}\right)^{1 / 3}+a, \\
\frac{\partial Q_{f}}{\partial x}(x, y) & =\frac{(x-a)^{2}}{2^{1 / 3}\left((x-a)^{3}+(y-a)^{3}\right)^{2 / 3}} .
\end{aligned}
$$

Thus it is not continuously differentiable at $(a, a)$. In fact, we cannot define the quasiweights

$$
\lim _{\varepsilon \rightarrow 0} \frac{\partial Q_{f}}{\partial x}(a+\varepsilon, a)=\lim _{\varepsilon \rightarrow 0} 2^{-1 / 3}=2^{-1 / 3},
$$


4 Continuously differentiable means

while

$$
\lim _{\varepsilon \rightarrow 0} \frac{\partial Q_{f}}{\partial x}(a+\varepsilon, a+\varepsilon)=\frac{1}{2^{1 / 3} \times 2^{2 / 3}}=\frac{1}{2} .
$$

Therefore, $Q_{f}$ is not a $C^{1}$-mean.

If a $C^{1}$-mean $M$ satisfies

$$
M\left(x_{\pi_{1}}, \ldots, x_{\pi_{n}}\right)=M\left(x_{1}, \ldots, x_{n}\right)
$$

for all permutation $\pi$, then it is called symmetric. It is clear that all quasi-weights for all symmetric $C^{1}$-means are the same value $1 / n$. But the converse is false by the following example.

Example 2.4. The first quasi-weight of the following arithmetic (resp., geometric) mean

$$
\left.A(a, b)=w_{1} a+w_{2} b \quad \text { (resp., } G(a, b)=a^{w_{2}} b^{w_{1}}\right)
$$

coincides with the first weight $w_{1}$ (resp., $\left.w_{2}\right)$. Putting $M(a, b)=(A(a, b)+G(a, b)) / 2$, we have

$$
w(M)_{1}(a)=w(M)_{2}(a)=\frac{w_{1}+w_{2}}{2}=\frac{1}{2},
$$

while $M(a, b)$ is not symmetric if $w_{1} \neq w_{2}$.

\section{Continuous differentiability}

Since functions $M_{f, P}$ include $Q_{f, w}$ as a case of singular measures, we should also assume that $f$ has no stationary points to discuss $\mathcal{M}_{f, P}$. So we consider a case of absolutely continuous measures with the Radon-Nikodym derivative $\phi_{x_{1}, \ldots, x_{n}}$ :

$$
M_{f, \phi}\left(x_{1}, \ldots, x_{n}\right)=f^{-1}\left(\int_{0}^{\infty} f(x) \phi_{x_{1}, \ldots, x_{n}}(x) d x\right) .
$$

The following example shows that we need the condition $\phi(x)>0$ for all $x>0$ in order that $M_{f, \phi}$ be a $C^{1}$-mean.

Example 3.1. Consider the derivative

$$
\phi_{a, b}(x)=\frac{3(x-1)^{2} \chi_{[a, b]}(x)}{(b-1)^{3}-(a-1)^{3}}
$$

(for convenience's sake, $\chi_{[a, b]}=-\chi_{[b, a]}$ if $a>b$ and $\phi_{a, a}(x)=a$ ). Then we have $\phi_{a, b}(1)=0$. Since

$$
M_{x, \phi}(a, b)=\frac{3}{(b-1)^{3}-(a-1)^{3}} \int_{a}^{b} x(x-1)^{2} d x=\frac{(1+3 b / 4)(b-1)^{3}-(1+3 a / 4)(a-1)^{3}}{(b-1)^{3}-(a-1)^{3}},
$$


it follows that $M_{x, \phi}(a, b)$ is symmetric. So quasi-weights are $1 / 2$ if it is a $C^{1}$-mean, while

$$
\lim _{\varepsilon \rightarrow 0} \frac{M_{x, \phi}(1+\varepsilon, 1)-M_{x, \phi}(1,1)}{\varepsilon}=\lim _{\varepsilon \rightarrow 0} \frac{1+(3 / 4) \varepsilon-1}{\varepsilon}=\frac{3}{4} .
$$

Now let $p$ be a polynomial with a degree $m>0$ which is monotone and convex on $(0, \infty)$. Then $p^{\prime}(x)>0$ for all $x>0$. For a continuously differentiable monotone function $f$ with no stationary points, we define

$$
M_{f, p}(a, b)=f^{-1}\left(\int_{a}^{b} \frac{f(x) p^{\prime}(x)}{p(b)-p(a)} d x\right)
$$

for $a \neq b$ and $M_{f, p}(a, a)=a$. We will prove that $M_{f, p}$ defined above is a $C^{1}$-mean. To do this, we first show the continuity of $M_{f, p}$.

Lemma 3.2. $M_{f, p}$ is continuous.

Proof. For the interval $I_{a, b}$ between $a$ and $b$, there exists $\xi_{a, b} \in I_{a, b}$ with

$$
\int_{a}^{b} f(t) p^{\prime}(t) d t=f\left(\xi_{a, b}\right) p^{\prime}\left(\xi_{a, b}\right)(b-a)
$$

by the mean value theorem. Moreover, Cauchy's mean-value theorem says that there exists $c_{a, b}$ with

$$
\frac{1}{p^{\prime}\left(c_{a, b}\right)}=\frac{b-a}{p(b)-p(a)}
$$

for $a \neq b$. Put $B_{\varepsilon}(c)=(c-\varepsilon, c+\varepsilon)$ for a fixed $c>0$. Then, for $a \neq b \in B_{\varepsilon}(c)$,

$$
M_{f, p}(a, b)=f^{-1}\left(\int_{a}^{b} \frac{f(x) p^{\prime}(x)}{p(b)-p(a)} d x\right)=f^{-1}\left(\frac{f\left(\xi_{a, b}\right) p^{\prime}\left(\xi_{a, b}\right)}{p^{\prime}\left(c_{a, b}\right)}\right),
$$

so that, as $\varepsilon \rightarrow 0$, we have $\xi_{a, b}, c_{a, b} \rightarrow c$ and $M_{f, p}(a, b)$ converges to

$$
f^{-1}\left(\frac{f(c) p^{\prime}(c)}{p^{\prime}(c)}\right)=c
$$

which implies $M_{f, p}$ is continuous.

To verify that $M_{f, p}$ is a $C^{1}$-mean, we first show that it satisfies (i).

Lemma 3.3. $M_{f, p}(a, b)$ is invariant for every affine transform of $f \mapsto t f+s(t \neq 0)$ and $M_{f, p}(a, b)$ is monotone increasing in each term. 
6 Continuously differentiable means

Proof. Since $(t f+s)^{-1}(x)=f^{-1}((1 / t)(x-s))$, we have

$$
\begin{aligned}
M_{t f+s, p}(a, b) & =(t f+s)^{-1}\left(\int_{a}^{b} \frac{(t f(x)+s) p^{\prime}(x)}{p(b)-p(a)} d x\right) \\
& =(t f+s)^{-1}\left(t \int_{a}^{b} \frac{f(x) p^{\prime}(x)}{p(b)-p(a)} d x+s \int_{a}^{b} \frac{p^{\prime}(x)}{p(b)-p(a)} d x\right) \\
& =(t f+s)^{-1}\left(t \int_{a}^{b} \frac{f(x) p^{\prime}(x)}{p(b)-p(a)} d x+s\right) \\
& =f^{-1}\left(\int_{a}^{b} \frac{f(x) p^{\prime}(x)}{p(b)-p(a)} d x\right)=M_{f, p}(a, b) .
\end{aligned}
$$

Thus we may assume that $f$ (and hence $f^{-1}$ ) is monotone increasing. Since

$$
\begin{aligned}
\frac{\partial}{\partial y} \int_{a}^{y} \frac{f(x) p^{\prime}(x)}{p(y)-p(a)} d x & =\frac{\left(f(y)(p(y)-p(a))-\int_{a}^{y} f(x) p^{\prime}(x) d x\right) p^{\prime}(y)}{(p(y)-p(a))^{2}} \\
& \geqq \frac{\left(f(y)(p(y)-p(a))-f(y) \int_{a}^{y} p^{\prime}(x) d x\right) p^{\prime}(y)}{(p(y)-p(a))^{2}}=0,
\end{aligned}
$$

we have $\int_{a}^{y}\left(f(x) p^{\prime}(x) /(p(y)-p(a))\right) d x$ is monotone increasing for $y$. Therefore, $M_{f, p}(a$, b) satisfies (i).

Next, to show the differentiability, we cite the following fundamental lemma (for the sake of completeness, here we give a proof).

Lemma 3.4. Let $g$ be a $C^{2}$-function on $(0, \infty)$ and

$$
G(x, y)= \begin{cases}\frac{g(y)-g(x)}{y-x} & \text { if } x \neq y, \\ g^{\prime}(x) & \text { if } x=y .\end{cases}
$$

Then $G$ is a $C^{1}$-function on $(0, \infty)^{2}$.

Proof. Since $G$ is symmetric, it suffices to show that $G_{x}$ is continuous at the diagonal set $\{(c, c) \mid c>0\}$. By the l'Hospital theorem,

$$
\lim _{h \rightarrow 0} \frac{G(c+h, c)-G(c, c)}{h}=\lim _{h \rightarrow 0} \frac{g(c+h)-g(c)-h g^{\prime}(c)}{h^{2}}=\lim _{h \rightarrow 0} \frac{g^{\prime}(c+h)-g^{\prime}(c)}{2 h}=\frac{g^{\prime \prime}(c)}{2}
$$


that is, $G_{x}(c, c)=g^{\prime \prime}(c) / 2$. Since $g^{\prime \prime}$ is continuous,

$$
\lim _{h \rightarrow 0} G_{x}(c+h, c+h)=\lim _{h \rightarrow 0} \frac{g^{\prime \prime}(c+h)}{2}=\frac{g^{\prime \prime}(c)}{2} .
$$

On the other hand, for $x \neq y$, we have

$$
G_{x}(x, y)=\frac{\partial}{\partial x}\left(\frac{g(y)-g(x)}{y-x}\right)=\frac{g(y)-g(x)-g^{\prime}(x)(y-x)}{(y-x)^{2}} .
$$

Now what we must show is $\lim _{h \neq k \rightarrow 0} G_{x}(c+h, c+k)=G_{x}(c, c)$. Let $h \neq k \in B_{\varepsilon}(0)$. By Taylor's expansion theorem, there exists $\xi_{h, k} \in I_{c+h, c+k} \subset B_{\varepsilon}(c)$ with

$$
g(c+h)=g(c+k)+g^{\prime}(c+k)(h-k)+\frac{1}{2} g^{\prime \prime}\left(\xi_{h, k}\right)(h-k)^{2} .
$$

The mean-value theorem says that there exists $c_{h, k} \in I_{c+h, c+k} \subset B_{\varepsilon}(c)$ with

$$
g^{\prime}(c+k)-g^{\prime}(c+h)=g^{\prime \prime}\left(c_{h, k} v\right)(k-h) .
$$

Thereby, as $\varepsilon \rightarrow 0$, we have $\xi_{h, k}, c_{h, k} \rightarrow c$ and

$$
\begin{aligned}
G_{x}(c+h, c+k) & =\frac{g(c+k)-g(c+h)-g^{\prime}(c+h)(k-h)}{(k-h)^{2}} \\
& =\frac{g^{\prime}(c+k)(k-h)-g^{\prime \prime}\left(\xi_{h, k}\right)(k-h)^{2} / 2-g^{\prime}(c+h)(k-h)}{(k-h)^{2}} \\
& =\frac{g^{\prime}(c+k)-g^{\prime}(c+h)}{k-h}-\frac{g^{\prime \prime}\left(\xi_{h, k}\right)}{2} \\
& =g^{\prime \prime}\left(c_{h, k}\right)-\frac{g^{\prime \prime}\left(\xi_{h, k}\right)}{2} \longrightarrow g^{\prime \prime}(c)-\frac{g^{\prime \prime}(c)}{2}=\frac{g^{\prime \prime}(c)}{2},
\end{aligned}
$$

which implies $G$ is continuously differentiable.

Now we have the following theorem.

Theorem 3.5. The above function $M_{f, p}(a, b)$ defines a symmetric $C^{1}$-mean.

Proof. Let $F_{k}$ be primitive functions defined inductively

$$
F_{k+1}^{\prime}=F_{k}, \quad F_{0}=f
$$


8 Continuously differentiable means

Since $p^{(m)}(x)$ is a constant function, we have

$$
\begin{aligned}
\int_{a}^{b} f(x) p^{\prime}(x) d x & =\left[F_{1}(x) p^{\prime}(x)\right]_{a}^{b}-\int_{a}^{b} F_{1}(x) p^{\prime \prime}(x) d x \\
& =F_{1}(b) p^{\prime}(b)-F_{1}(a) p^{\prime}(a)-\left[F_{2}(x) p^{\prime \prime}(x)\right]_{a}^{b}+\int_{a}^{b} F_{2}(x) p^{(3)}(x) d x \\
& =\cdots \\
& =\sum_{k=1}^{m-1}(-1)^{k+1}\left(F_{k}(b) p^{(k)}(b)-F_{k}(a) p^{(k)}(a)\right)+(-1)^{m+1} \int_{a}^{b} F_{m-1}(x) p^{(m)}(x) d x \\
& =\sum_{k=1}^{m}(-1)^{k+1}\left(F_{k}(b) p^{(k)}(b)-F_{k}(a) p^{(k)}(a)\right) .
\end{aligned}
$$

It follows that

$$
M_{f, p}(a, b)=f^{-1}\left(\frac{\sum_{k=1}^{m}(-1)^{k+1}\left(F_{k}(b) p^{(k)}(b)-F_{k}(a) p^{(k)}(a)\right)}{p(b)-p(a)}\right) .
$$

Here we put

$$
H_{k}(a, b) \equiv \frac{F_{k}(b) p^{(k)}(b)-F_{k}(a) p^{(k)}(a)}{p(b)-p(a)} .
$$

Note that a polynomial $P(a, b)=(p(b)-p(a)) /(b-a)$ no longer have a divisor $b-a$ by $p^{\prime}(x)>0$, and hence $P(a, b) \neq 0$ for all $a, b>0$. By Lemma 3.3, $P$ is continuously differentiable (by putting $\left.P(a, a) \equiv p^{\prime}(a)\right)$. Setting such functions

$$
P[k](a, b)=\frac{p^{(k)}(b)-p^{(k)}(a)}{b-a}, \quad G[k](a, b)=\frac{F_{k}(b)-F_{k}(a)}{b-a}
$$

(where $\left.P[k](a, a) \equiv p^{(k+1)}(a), G[k](a, a) \equiv F_{k-1}(a)\right)$, we have

$$
H_{k}(a, b)=\frac{F_{k}(b) P[k](a, b)+G[k](a, b) p^{(k)}(a)}{P(a, b)},
$$

and hence it is continuously differentiable by Lemma 3.3. Therefore, since $f^{-1}$ is also continuously differentiable, $M_{f, p}(a, b)$ is a symmetric $C^{1}$-mean by Lemma 3.2.

\section{Skew power means}

For positive numbers $a$ and $b$, consider the following special case for $M_{f, \phi}$ (see [2]):

$$
M_{t}(a, b)=\left(\frac{1}{b-a} \int_{a}^{b} x^{t} d x\right)^{1 / t}=\left(\frac{b^{t+1}-a^{t+1}}{(t+1)(b-a)}\right)^{1 / t} .
$$


Table 4.1

\begin{tabular}{c|ccccccc}
\hline $\mathrm{t}$ & $-\infty$ & -2 & -1 & 0 & 1 & 2 & $\infty$ \\
\hline & Minimum & Geometric & Logarithmic & Identric & Arithmetic & - & Maximum \\
& $\min \{a, b\}$ & $\sqrt{a b}$ & $\frac{b-a}{\log b-\log a}$ & $\frac{a^{a /(a-b)} b^{b /(b-a)}}{e}$ & $\frac{a+b}{2}$ & $\left(\frac{a^{2}+a b+b^{2}}{3}\right)^{1 / 2}$ & $\max \{a, b\}$ \\
\hline
\end{tabular}

By definition, we call them skew power means which include various classical means, for example, the logarithmic and identric ones as shown in Table 4.1.

Here we use 2-variable power means

$$
P_{r, w}(a, b)=\left((1-w) a^{r}+w b^{r}\right)^{1 / r}
$$

and, in particular,

$$
P_{0, w}(a, b) \equiv \lim _{r \rightarrow 0} P_{r, w}(a, b)=a^{1-w} b^{w}
$$

is a weighted geometric mean. For the convenience's sake, we omit $w$ for $w=1 / 2$ which is a symmetric mean.

Let $\alpha_{n}$ be the smallest number $N$ such that $M_{n}$ is a composition of $N$ power means $\left(\alpha_{n}=\infty\right.$ if not). Putting

$$
L_{n, m}(a, b)=\left(M_{n}\left(a^{m}, b^{m}\right)\right)^{1 / m}
$$

we define $\beta_{n, m}$ as the smallest number $N$ such that $L_{n, m}$ is a composition of $N$ power means $\left(\beta_{n, m}=\infty\right.$ if not). Now we have the following theorem.

THeOREM 4.1. The mean function $M_{n}(a, b)$ is represented by a composition for finitely many power means as follows.

(i) For all positive integers $n$, it is a composition of at most $2 n-1$ power means.

(ii) For all integers $n \leqq-2$, it is a composition of at most $-2 n-3$ power means.

Proof

Case 1. Let $n$ be a fixed positive integer and $k$ a fixed nonzero integer. By $M_{1}=P_{1}$, we have

$$
\alpha_{1}=1
$$

and also

$$
\alpha_{2} \leqq 3
$$


by

$$
M_{2}(a, b)=\left(\frac{a^{2}+a b+b^{2}}{3}\right)^{1 / 2}=\left(\frac{2}{3} \frac{a^{2}+b^{2}}{2}+\frac{1}{3} a b\right)^{1 / 2}=P_{2,1 / 3}\left(P_{2}(a, b), P_{0}(a, b)\right) .
$$

Since

$$
\begin{aligned}
M_{2 n+1}(a, b) & =\left(\frac{a^{2 n+1}+a^{2 n} b+\cdots+b^{2 n+1}}{2 n+2}\right)^{1 /(2 n+1)}=\left(\frac{a^{n}+\cdots+b^{n}}{n+1} \frac{a^{n+1}+b^{n+1}}{2}\right)^{1 /(2 n+1)} \\
& =M_{n}(a, b)^{n /(2 n+1)} P_{n+1}(a, b)^{(n+1) /(2 n+1)}=P_{0,(n+1) /(2 n+1)}\left(M_{n}(a, b), P_{n+1}(a, b)\right),
\end{aligned}
$$

we have

$$
\alpha_{2 n+1} \leqq \alpha_{n}+2
$$

Moreover,

$$
\alpha_{2 n} \leqq \beta_{n, 2}+\beta_{n-1,2}+3,
$$

since

$$
\begin{aligned}
& M_{2 n}(a, b)=\left(\frac{a^{2 n}+a^{2 n-1} b+\cdots+b^{2 n}}{2 n+1}\right)^{1 / 2 n} \\
& =\left(\frac{a^{2 n}+a^{2(n-1)} b^{2}+\cdots+b^{2 n}+\left(a^{2 n-2}+a^{2 n-4} b^{2}+\cdots+b^{2 n-2}\right) a b}{2 n+1}\right)^{1 / 2 n} \\
& =\left(\frac{(n+1) M_{n}\left(a^{2}, b^{2}\right)^{n}+n M_{n-1}\left(a^{2}, b^{2}\right)^{n-1} a b}{2 n+1}\right)^{1 / 2 n} \\
& =P_{2 n, n /(2 n+1)}\left(\sqrt{M_{n}\left(a^{2}, b^{2}\right)}, P_{0,1 / n}\left(\sqrt{M_{n-1}\left(a^{2}, b^{2}\right)}, \sqrt{a b}\right)\right) \\
& =P_{2 n, n /(2 n+1)}\left(L_{n, 2}(a, b), P_{0,1 / n}\left(\sqrt{M_{n-1}\left(a^{2}, b^{2}\right)}, \sqrt{a b}\right)\right) \\
& =P_{2 n, n /(2 n+1)}\left(L_{n, 2}(a, b), P_{0,1 / n}\left(L_{n-1,2}(a, b), P_{0}(a, b)\right)\right) .
\end{aligned}
$$

It follows from $L_{1,2 k}(a, b)=M_{1}\left(a^{2 k}, b^{2 k}\right)^{1 /(2 k)}=P_{2 k}(a, b)$ that

$$
\beta_{1,2 k}=1 \text {. }
$$

Moreover we have

$$
\beta_{2,2 k} \leqq 3
$$


by

$$
L_{2,2 k}(a, b)=M_{2}\left(a^{2 k}, b^{2 k}\right)^{1 / 2 k}=\left(\frac{a^{4 k}+(a b)^{2 k}+b^{4 k}}{3}\right)^{1 / 4 k}=P_{4 k, 1 / 3}\left(P_{4 k}(a, b), P_{0}(a, b)\right) .
$$

Since

$$
\begin{aligned}
L_{2 n+1,2 k}(a, b) & =M_{2 n+1}\left(a^{2 k}, b^{2 k}\right)^{1 / 2 k} \\
& =P_{0,(n+1) /(2 n+1)}\left(M_{n}\left(a^{2 k}, b^{2 k}\right), P_{n+1}\left(a^{2 k}, b^{2 k}\right)\right)^{1 / 2 k} \text { by }(4.8) \\
& =P_{0,(n+1) /(2 n+1)}\left(M_{n}\left(a^{2 k}, b^{2 k}\right)^{1 / 2 k}, P_{n+1}\left(a^{2 k}, b^{2 k}\right)^{1 / 2 k}\right) \\
& =P_{0,(n+1) /(2 n+1)}\left(L_{n, 2 k}(a, b), P_{2 k(n+1)}(a, b)\right)
\end{aligned}
$$

we have

$$
\beta_{2 n+1,2 k} \leqq \beta_{n, 2 k}+2
$$

Also we have

$$
\beta_{2 n, 2 k} \leqq \beta_{n, 4 k}+\beta_{n-1,4 k}+3
$$

by the following relation:

$$
\begin{aligned}
L_{2 n, 2 k}(a, b) & =M_{2 n}\left(a^{2 k}, b^{2 k}\right)^{1 / 2 k} \\
& =P_{2 n, n /(2 n+1)}\left(\sqrt{M_{n}\left(a^{4 k}, b^{4 k}\right)}, P_{0,1 / n}\left(\sqrt{M_{n-1}\left(a^{4 k}, b^{4 k}\right)}, \sqrt{a^{2 k} b^{2 k}}\right)\right)^{1 / 2 k} \quad(b y(4.11)) \\
& =\left(\frac{n+1}{2 n+1} \sqrt{M_{n}\left(a^{4 k}, b^{4 k}\right)}+\frac{n}{2 n+1} P_{0,1 / n}\left(\sqrt{M_{n-1}\left(a^{4 k}, b^{4 k}\right)}, a^{k} b^{k}\right)^{2 n}\right)^{1 / 4 k n} \\
& =\left(\frac{n+1}{2 n+1} M_{n}\left(a^{4 k}, b^{4 k}\right)^{4 k n / 4 k}+\frac{n}{2 n+1} P_{0,1 / n}\left(\sqrt{M_{n-1}\left(a^{4 k}, b^{4 k}\right)}, a^{k} b^{k}\right)^{4 k n / 2 k}\right)^{1 / 4 k n} \\
& =P_{4 k n, n /(2 n+1)}\left(L_{n, 4 k}(a, b), P_{0,1 / n}\left(\sqrt{\left.M_{n-1}\left(a^{4 k}, b^{4 k}\right), a^{k} b^{k}\right)^{1 / 2 k}}\right)\right. \\
& =P_{4 k n, n /(2 n+1)}\left(L_{n, 4 k}(a, b), P_{0,1 / n}\left(M_{n-1}\left(a^{4 k}, b^{4 k}\right)^{1 / 4 k}, \sqrt{a b}\right)\right) \\
& =P_{4 k n, n /(2 n+1)}\left(L_{n, 4 k}(a, b), P_{0,1 / n}\left(L_{n-1,4 k}(a, b), P_{0}(a, b)\right)\right) .
\end{aligned}
$$

Now, we show that

$$
A(n): \beta_{n, 2 k} \leqq 2 n-1 \quad(\forall k \in \mathbb{Z} \backslash\{0\})
$$


holds for all positive integers $n$. In fact, both $A(1)$ and $A(2)$ are true by (4.12) and (4.13). Assume that $A(n)$ holds for all $1 \leqq n \leqq N(N \geqq 2)$. If $N=2 \ell$, then

$$
\beta_{N+1,2 k}=\beta_{2 \ell+1,2 k} \leqq \beta_{\ell, 2 k}+2 \leqq 2 \ell-1+2=N+1 \leqq 2(N+1)-1
$$

holds by (4.16) and the assumption, so that $A(N+1)$ holds. Also if $N=2 \ell+1$, then

$$
\beta_{N+1,2 k}=\beta_{2(\ell+1), 2 k} \leqq \beta_{\ell+1,4 k}+\beta_{\ell, 4 k}+3 \leqq 2(\ell+1)-1+2 \ell-1+3=4 \ell+3=2(N+1)-1
$$

holds by (4.17) and the assumption, so that $A(N+1)$ holds. Therefore, $A(n)$ holds for all positive integers $n$ by induction. We next show that the required inequality

$$
B(n): \alpha_{n} \leqq 2 n-1
$$

holds for all positive integers $n$. In fact, both $B(1)$ and $B(2)$ are true by (4.5) and (4.6). Assume that $B(n)$ holds for all $1 \leqq n \leqq N(N \geqq 2)$. If $N=2 \ell$, then

$$
\alpha_{N+1}=\alpha_{2 \ell+1} \leqq \alpha_{\ell}+2 \leqq 2 \ell-1+2=N+1 \leqq 2(N+1)-1
$$

holds by (4.9) and the assumption, and hence $B(N+1)$ holds. Also if $N=2 \ell+1$, then

$$
\alpha_{N+1}=\alpha_{2(\ell+1)} \leqq \beta_{\ell+1,2}+\beta_{\ell, 2}+3 \leqq 2(\ell+1)-1+2 \ell-1+3=4 \ell+3=2(N+1)-1
$$

holds by (4.10), $A(n)$, and the assumption, so that $B(N+1)$ holds. Therefore, $B(n)$ holds for all positive integers $n$ by induction. Thus we have Case 1 .

Case 2. Let $n$ be a fixed positive integer. By $M_{-2}=P_{0}$, we have

$$
\alpha_{-2}=1
$$

It follows from $L_{1,-1}=P_{1}$ that

$$
\beta_{1,-1}=1
$$

Since

$$
L_{2,-1}(a, b)=M_{2}\left(\frac{1}{a}, \frac{1}{b}\right)^{-1}=\left(\frac{a^{-2}+(a b)^{-1}+b^{-2}}{3}\right)=P_{-2,1 / 3}\left(P_{-2}(a, b), P_{0}(a, b)\right),
$$

we have

$$
\beta_{2,-1} \leqq 3
$$

Moreover, we have

$$
\beta_{2 n+1,-1} \leqq \beta_{n,-1}+2
$$


by the following equation:

$$
\begin{aligned}
L_{2 n+1,-1}(a, b) & =M_{2 n+1}\left(\frac{1}{a}, \frac{1}{b}\right)^{-1} \\
& =\left(M_{n}\left(\frac{1}{a}, \frac{1}{b}\right)^{-1}\right)^{n /(2 n+1)} P_{-(n+1)}(a, b)^{(n+1) /(2 n+1)} \quad \text { by }(4.8) \\
& =P_{0,(n+1) /(2 n+1)}\left(L_{n,-1}(a, b), P_{-(n+1)}(a, b)\right) .
\end{aligned}
$$

Since

$$
\begin{aligned}
L_{2 n,-1}(a, b) & =M_{2 n}\left(\frac{1}{a}, \frac{1}{b}\right)^{-1} \\
& =P_{2 n, n /(2 n+1)}\left(L_{n, 2}\left(\frac{1}{a}, \frac{1}{b}\right), P_{0,1 / n}\left(L_{n-1,2}\left(\frac{1}{a}, \frac{1}{b}\right), P_{0}\left(\frac{1}{a}, \frac{1}{b}\right)\right)\right)^{-1} \\
& =\left(\frac{n+1}{2 n+1} L_{n, 2}\left(\frac{1}{a}, \frac{1}{b}\right)^{2 n}+\frac{n}{2 n+1} P_{0,1 / n}\left(L_{n-1,2}\left(\frac{1}{a}, \frac{1}{b}\right), P_{0}\left(\frac{1}{a}, \frac{1}{b}\right)\right)^{2 n}\right)^{-1 / 2 n} \\
& =\left(\frac{n+1}{2 n+1} L_{n,-2}(a, b)^{-2 n}+\frac{n}{2 n+1}\left(\left(M_{n-1}\left(a^{-2}, b^{-2}\right)\right)^{(n-1) / 2 n}(a b)^{-1 / 2 n}\right)^{2 n}\right)^{-1 / 2 n} \\
& =\left(\frac{n+1}{2 n+1} L_{n,-2}(a, b)^{-2 n}+\frac{n}{2 n+1}\left(\left(M_{n-1}\left(a^{-2}, b^{-2}\right)\right)^{-(n-1) / 2 n} P_{0}(a, b)^{1 / n}\right)^{-2 n}\right)^{-1 / 2 n} \\
& =\left(\frac{n+1}{2 n+1} L_{n,-2}(a, b)^{-2 n}+\frac{n}{2 n+1}\left(\left(L_{n-1,-2}(a, b)\right)^{(n-1) / n} P_{0}(a, b)^{1 / n}\right)^{-2 n}\right)^{-1 / 2 n} \\
& =P_{-2 n, n /(2 n+1)}\left(L_{n,-2}(a, b), P_{0,1 / n}\left(L_{n-1,-2}(a, b), P_{0}(a, b)\right)\right),
\end{aligned}
$$

it follows from $A(n)$ that

$$
\beta_{2 n,-1} \leqq \beta_{n,-2}+\beta_{n-1,-2}+3 \leqq 2 n-1+2(n-1)-1+3=4 n-1 .
$$

Then we have

$$
\alpha_{-(n+2)} \leqq \beta_{n,-1}+2
$$


since

$$
\begin{aligned}
M_{-(n+2)}(a, b) & =\left(\frac{b^{-(n+1)}-a^{-(n+1)}}{-(n+1)(b-a)}\right)^{-1 /(n+2)}=\left(\frac{b^{-(n+1)}-a^{-(n+1)}}{(n+1)(1 / b-1 / a) a b}\right)^{-1 /(n+2)} \\
& =\left(\frac{(1 / a)^{n}+(1 / a)^{n-1}(1 / b)+\cdots+(1 / b)^{n}}{n+1}\right)^{-1 /(n+2)}(a b)^{1 /(n+2)} \\
& =\left(M_{n}\left(\frac{1}{a}, \frac{1}{b}\right)^{-1}\right)^{n /(n+2)} \sqrt{a b}^{2 /(n+2)}=P_{0,2 /(n+2)}\left(L_{n,-1}(a, b), P_{0}(a, b)\right) .
\end{aligned}
$$

Now, we show that

$$
C(n): \beta_{n,-1} \leqq 2 n-1
$$

holds for all positive integers $n$. In fact, both $C(1)$ and $C(2)$ are true by (4.26) and (4.28). Assume that $C(n)$ holds for all $1 \leqq n \leqq N(N \geqq 2)$. If $N=2 \ell$, then

$$
\beta_{N+1,-1}=\beta_{2 \ell+1,-1} \leqq \beta_{\ell,-1}+2 \leqq 2 \ell-1+2=N+1 \leqq 2(N+1)-1
$$

holds by (4.29) and the assumption, so that $C(N+1)$ holds. Also if $N=2 \ell+1$, then

$$
\beta_{N+1,-1}=\beta_{2(\ell+1),-1} \leqq 4(\ell+1)-1=4 \ell+3=2(N+1)-1
$$

holds by (4.32) and the assumption, so that $C(N+1)$ holds. Therefore, $C(n)$ holds for all positive integers $n$ by induction. Thereby, let $m$ be an integer with $m \leqq-2$. If $m \leqq-3$, then $1 \leqq-m-2$, and hence

$$
\alpha_{m}=\alpha_{-(-m-2+2)} \leqq \beta_{-m-2,-1}+2 \leqq 2(-m-2)-1+2=-2 m-3
$$

by $C(n)$. Also if $m=-2$, then the above inequality holds by (4.25). Thus we have Case 2 , which completes the proof.

Finally we conjecture that the above relations hold for all rational numbers $t \neq 0,-1$.

\section{Acknowledgments}

The authors would like to thank the referees for the careful reading and useful suggestions which improved the paper. The authors also thank Fumio Hiai and Shizuo Miyajima for their valuable comments. The fourth and fifth authors are partially supported by the Grants-in-Aid for Scientific Research, Japan Society for the Promotion of Science, Japan.

\section{References}

[1] J. I. Fujii, Kubo-Ando theory for convex functional means, Scientiae Mathematicae Japonicae 57 (2003), no. 2, 351-363.

[2] J. I. Fujii, M. Nakamura, and S.-E. Takahasi, Cooper's approach to chaotic operator means, Scientiae Mathematicae Japonicae 63 (2006), no. 2, 319-324.

[3] T. Furuta, J. Mićić, J. E. Pečarić, and Y. Seo, Mond-Pečarić Method in Operator Inequalities, Monographs in Inequalities, vol. 1, Element, Zagreb, 2005. 
[4] G. H. Hardy, J. E. Littlewood, and G. Pólya, Inequalities, Cambridge University Press, Cambridge, 1934, (2nd ed. 1951).

[5] T. Ito and C. Nara, Quasi-arithmetic means of continuous functions, Journal of the Mathematical Society of Japan 38 (1986), no. 4, 697-720.

[6] F. Kubo and T. Ando, Means of positive linear operators, Mathematische Annalen 246 (1980), no. 3, 205-224.

Jun Ichi Fujii: Information Science Division, Department of Arts and Sciences, Osaka Kyoiku University, Asahigaoka, Kashiwara, Osaka 582-8582, Japan

E-mail address: fujii@cc.osaka-kyoiku.ac.jp

Masatoshi Fujii: Department of Mathematics, Osaka Kyoiku University, Asahigaoka,

Kashiwara, Osaka 582-8582, Japan

E-mail address: mfujii@cc.osaka-kyoiku.ac.jp

Takeshi Miura: Group of Applied Mathematics and Physics, Department of Basic Technology, Yamagata University, Yonezawa 992-8510, Japan

E-mail address: miura@yz.yamagata-u.ac.jp

Hiroyuki Takagi: Department of Mathematics, Shinshu University, Asahi, Matsumoto, Nagano 390-8621, Japan

E-mail address: takagi@math.shinshu-u.ac.jp

Sin-Ei Takahasi: Group of Applied Mathematics and Physics, Department of Basic Technology, Yamagata University, Yonezawa 992-8510, Japan

E-mail address: sin-ei@emperor.yz.yamagata-u.ac.jp 